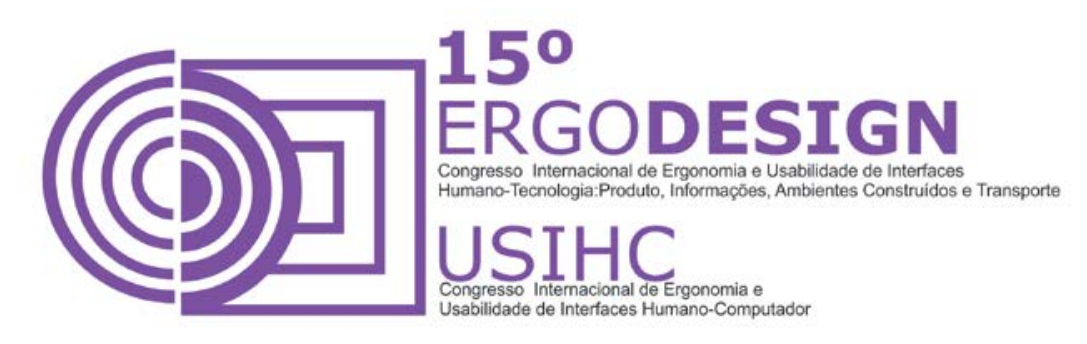

\title{
DESIGN E TECNOLOGIA ASSISTIVA: CONTRIBUIÇÕES PARA O DESENVOLVIMENTO DE CADEIRAS DE RODAS INTELIGENTES
}

\author{
SCATOLIM, Roberta Scatolim (1); \\ SANTOS, João Eduardo Guarnetti dos (2); \\ LANDIM, Paula da Cruz (3); \\ CASARI, Odair (4) \\ (1) UNESP/FAAC, Doutoranda \\ e-mail: robertascatolim@gmail.com \\ (2) UNESP/FAAC, Livre-docente \\ e-mail: guarneti@feb.unesp.br \\ (3) UNESP/FAAC, Livre-docente \\ e-mail: paula@faac.unesp.br \\ (4) UNIARA, Graduação \\ e-mail: odair@camarajaboticabal.sp.gov.br
}

\begin{abstract}
RESUMO
A acessibilidade é um direito, mas ainda são necessárias pesquisas e desenvolvimento de objetos que propiciem aos deficientes físicos a realização de atividades sem dificuldade. O objetivo é mostrar como a Inteligência Artificial e as Cadeiras de Rodas Inteligentes têm beneficiado aqueles que possuem mobilidades reduzidas, enfatizando o sistema Arduino, que possibilita o aprimoramento de tecnologias assistivas, com o desenvolvimento de objetos interativos e recursos que capazes de trazer mais qualidade de vida ao portador de lesão medular, com independência e segurança em seu cotidiano, além de contribuir para um design acessível, sem restrição de uso.
\end{abstract}

\begin{abstract}
Accessibility is a right, but are still necessary research and development objects that provide the disabled carrying out activities without difficulty. The aim is to show how Artificial Intelligence and Intelligent Wheelchairs have benefited those with reduced mobility, emphasizing the Arduino system, which allows the improvement of assistive technologies, with the development of interactive objects and resources able to bring more quality life for patients with spinal cord injury, with independence and security in their daily
\end{abstract}




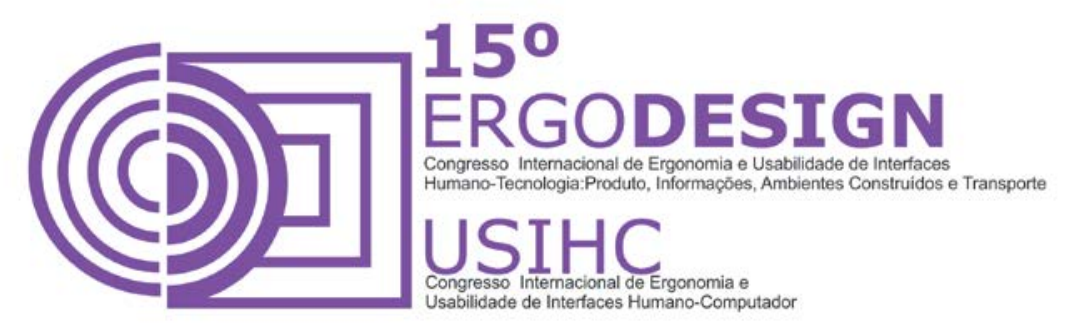

lives, and contribute to an affordable design, without restriction of use.

\section{INTRODUÇÃO}

O design pode diminuir dificuldades de mobilidade dos deficientes físicos por meio de vários recursos e ferramentas assistivas. A cadeira de rodas é resultado do desenvolvimento das tecnologias assistivas, que propiciam mais qualidade de vida, independência e segurança aos portadores de lesão medular ou com capacidades reduzidas.

Um dos maiores problemas enfrentados pelos portadores de lesão medular é o sedentarismo. As consequências motoras da lesão cervical determinam no comprometimento mais importante: a perda da capacidade de locomoção e o auxílio da cadeira de rodas.

Segundo Antoneli (2003), a cadeira de rodas permite o auxílio de deslocamento, constituindo uma das tecnologias assistivas usadas por pessoas com impossibilidade de locomoção, seja a deficiência temporária ou definitiva. Um equipamento que para ser utilizado necessita de prescrição de um profissional habilitado, para orientar de acordo com as necessidades do paciente.

De acordo com o Censo Demográfico 2010 do IBGE - Instituto Brasileiro de Geografia e Estatística, mais de 45,6 milhões de brasileiros declararam ter alguma deficiência, representando $23,9 \%$ da população do país. Um número relevante de usuários que possuem limitações para realizar tarefas cotidianas, como andar. Uma situação que exige adaptações e produtos acessíveis.

As lesões físicas geralmente são consequências de acidentes, que afetam as capacidades motoras. A lesão medular provoca mudanças corporais e sintomas que podem: diminuir a capacidade respiratória, pois os músculos intercostais começam a apresentar paresia; compromete os músculos do tronco, dos braços e das pernas; influi na perda da preensão dos dedos das mãos; disfunção da atividade da bexiga e do intestino, que exige o uso de fraldas e/ou de sondas; escaras (úlceras); infecções urinárias, que podem provocar insuficiências renais; disfunção do sistema de regulação térmica; comprometimentos da sexualidade; depressão, entre outros sintomas.

Nesse contexto, cadeira de rodas motorizada age como um recurso da Tecnologia Assistiva que determina da independência de indivíduos com mobilidade reduzida, quando adequada aos diversos níveis e necessidades dos usuários.

Percebemos o crescente aumento de atividades sociais dos portadores de lesão medular. $O$ mercado de trabalho, apesar de ainda restrito, mas regimentalmente é obrigado a incluir deficientes. Além disso, um cadeirante tem a vida social mais ativa, ao contrário de alguns anos, que pela falta de recursos, limitava-o ao sedentarismo e a rotina.

Nesse estudo enfatizaremos os portadores de paraplegia, quando a lesão compromete os membros inferiores, abaixo da cintura. Quanto mais baixo for o local da lesão na coluna 


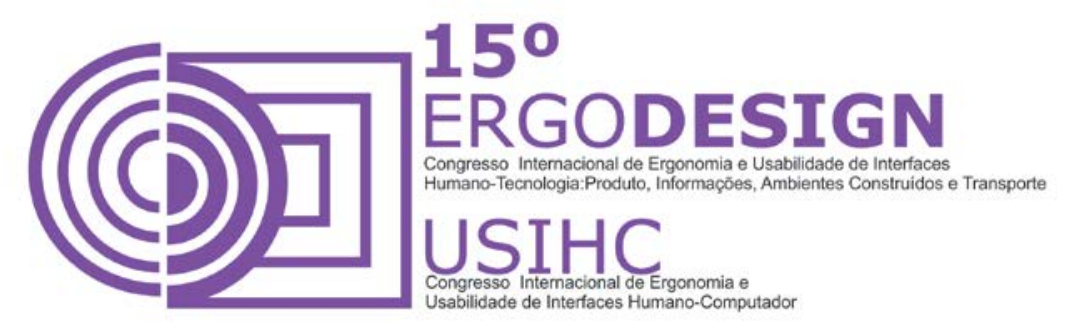

cervical, menores serão as consequências.

O desenvolvimento de novas tecnologias reduz problemas de mobilidade, uma relevante solução para pessoas portadoras de lesão medular. Determinante, de forma crescente, quantitativa e qualitativamente, nos sistemas de produção e tecnologias assistivas. Nesse contexto, o design ergonômico é um segmento do projeto de produto ou serviço com o intuito de garantir produtos eficientes e seguros.

Ergonomia, de acordo com lida (1990), é o estudo da adaptação do trabalho ao homem, que implica no ambiente físico (máquinas e equipamentos) e a relação do homem durante a atividade. Essa afirmação envolve várias áreas, com o objetivo de garantir o bem estar do homem na realização de uma tarefa, e suas consequências, como a prevenção de acidentes e a produtividade.

Dessa forma, esse estudo busca entender os benefícios que a cadeira de rodas inteligente traz aos portadores de lesão medular, enfatizando o Sistema Arduino, utilizado para desenvolver uma interação independente ou conectada ao computador. Esse sistema possui uma placa única e um grupo de softwares para programá-lo.

\section{CADEIRA DE RODAS INTELIGENTES (CRI)}

A partir da década de 1960, a Inteligência Artificial (IA) passou a ser aplicada em diversos segmentos industriais, voltada principalmente à área de elaboração de projetos e a avaliação de produtos industriais ou processos, devido, sobretudo, ao desenvolvimento e avanços na informática, ao uso crescente de computadores pessoais e internet, e a ampliação das práticas de técnicas de inteligência artificial (REZENDE, 2005).

De acordo com Bittencourt (2006) e Russel e Norvig (2004) Inteligência Artificial possui três linhas de pesquisa, subdivididas em:

- Conexionista: atua por meio de redes neurais artificiais, com a modelagem da inteligência humana e a simulação de integrantes do cérebro (neurônios e interligações);

- Simbólica: aplica a lógica para avaliar o comportamento inteligente através da linguagem. Pesquisa a aprendizagem, a representação e a concepção do conhecimento;

- Evolutiva: propõe a observação de mecanismos como a auto-organização e o comportamento adaptativo.

Dessa forma IA pode ser entendida como a inteligência humana mediada por mecanismos ou softwares, efetivada por pesquisas de computação que procuram métodos capazes de ampliar a capacidade racional para resolver problemas.

Löbach (2001) afirma que cadeiras de rodas são objetos de uso desenvolvidos para solucionar tensões, durante o processo de uso, advinda das necessidades. Tais objetos uso estão intrínsecos às condições de uma sociedade. Nesse contexto, para o autor os produtos industriais são objetos destinados a suprir necessidades, produzidos em larga escala, para um alto número de usuários. Cadeiras de rodas são sistemas composto basicamente por uma 


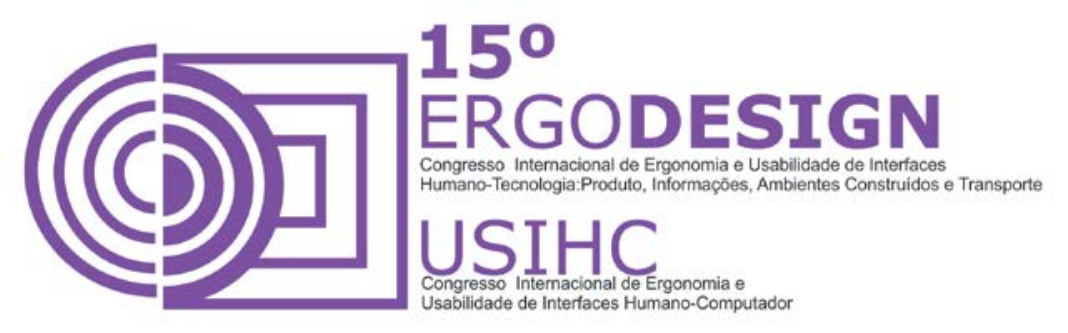

cadeira disposta em um par de rodas. É utilizada como dispositivo de locomoção para portadores de deficiência ou que tenham alguma mobilidade física. Basicamente dividem-se em duas categorias: manuais e eletrônica, ambos podem ser movidos manualmente pelo usuário ou empurradas por alguém. Fatores como o dimensional do encosto e do assento de acordo com as medidas antropométricas são indispensáveis para o bom desenvolvimento projetual de tal sistema. (SANTOS, 2013, p. 125)

A Cadeira de Rodas Inteligente utiliza uma tecnologia definida como um dispositivo robótico desenvolvido por meio da cadeira de rodas motorizada, de sistemas sensoriais e dispositivos, tornando-se uma ferramenta essencial para locomoção dos portadores de lesão medular ou com mobilidade reduzida.

Esse equipamento é utilizado em hospitais e domicílios e tem importante contribuição para os usuários que necessitam, proporcionando em suas atividades diárias mais segurança e independência.

As CRIs podem ser desenvolvidas com o uso de hardware e dispositivos específicos. São essenciais àqueles que têm limitações em utilizar uma cadeira de rodas convencional. Construídas para proporcionar segurança e facilitar a mobilidade dos cadeirantes, devido ao recurso semiautomático ou automático.

Os usuários das CRIs têm a praticidade em executar os movimentos por comandos, pelo apoio de dispositivos de entrada através da voz, expressões faciais, joystick, teclados, movimentos da cabeça e gestos, como podemos observar na Figura 1.

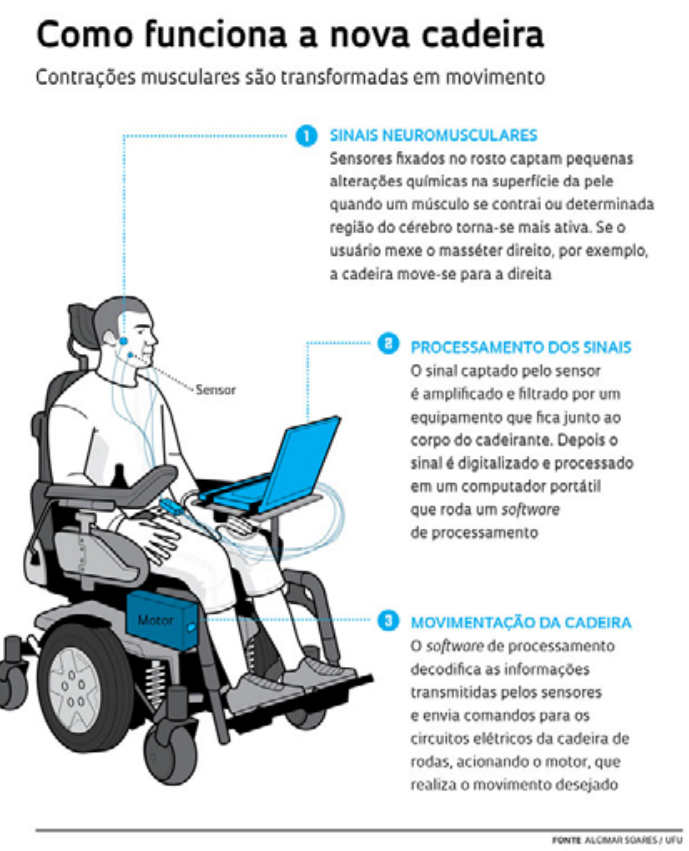

Figura 1. Funcionamento da Cadeira de Rodas Inteligente. Fonte: Revista FAPESP. 


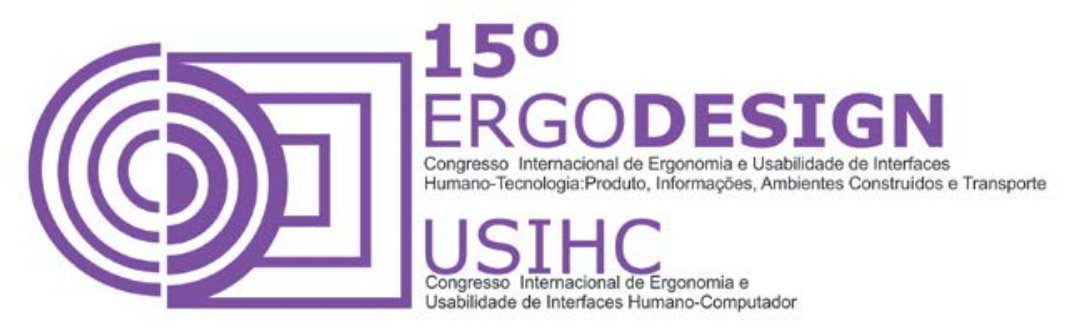

Conforme mostra a Figura 1, a CRI possui recursos que são ativados por contrações musculares. Os sinais neuromusculares são ativados por sensores que captam na superfície da pele a contração do músculo. Esses sinais são processados em um computador portátil que possui o software que decodifica as informações enviando comandos para a cadeira.

\subsection{Tecnologias Assistivas e sistemas de interação homem-máquina}

O desenvolvimento de interfaces homem-máquina nos projetos de CRI permitem que o usuário comande de forma abstrata, se comparado a interface com as cadeiras de rodas elétricas tradicionais. Como na usabilidade por meio do reconhecimento da face, cujo comando é determinado pela sequência de expressões.

Tecnologia Assistiva (TA) foi definida nos Estados Unidos em 1988, por meio de uma lei pública Technology-Related Assistance for Individuals with Disabilities Act - Public 100-407, como: qualquer produto ou sistema de produtos, adquiridos comercialmente, modificados, construído com adaptações, utilizado para ampliar, manter ou melhorar as habilidades do sujeito com limitações funcionais (MELLO, 1997).

Mello (1997), afirma que a tecnologia é considerada assistiva quando é capaz de auxiliar o desempenho funcional e reduzir incapacidades de atividades diárias. Difere-se de tecnologia reabilitadora, utilizada para recuperar de movimentos diminuídos.

TA implica o objeto (equipamento), a tecnologia concreta (prática) e o conhecimento exigido no processo de desenvolvimento, pesquisa, avaliação e criação da tecnologia.

Envolve várias áreas de aplicação: adaptações para atividades cotidianas; sistemas de comunicação alternativa; dispositivos para o uso de computadores; adequações em vários ambientes dos usuários; adaptação às necessidades, costumes e limitações (deficiências); mobilidade e acessibilidade.

A diversidade de deficiências ou incapacidades acaba levando o sujeito portador da lesão a situações afins: a segregação e a exclusão social.

Os recursos tecnológicos são definidos por Tecnologia Assistiva, Tecnologia de Assistência, Tecnologia de Apoio e Tecnologia de Ajuda. Tais conceitos devem proporcionar ao usuário, além do simples acesso aos equipamentos, condições que vão além da usabilidade, através de pesquisas e recursos que visam garantir a autonomia e reduzir a exclusão social.

Dessa forma, podemos afirmar que o design ergonômico envolve princípios de usabilidade, ergonomia e design, fundamentado por metodologias que primam por melhorar 0 desenvolvimento de produtos, através da interação entre o homem e dispositivos tecnológicos a satisfação de necessidades e aspirações tem um papel substancial, motivando a criação e o aperfeiçoamento de objetos. (LÖBACH, 2001, p. 29).

Jordan (1998) afirma que usabilidade não se limita mais em tecnologia da informação, é aplicada em outras áreas, devido a sua importância em garantir uso com segurança, qualidade e sem limitação. 


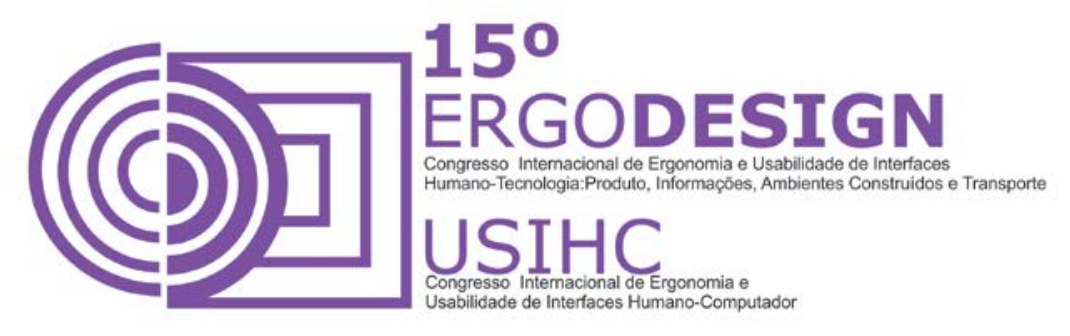

O design, independente das limitações do usuário, é um mediador da usabilidade, acessibilidade e principalmente da inclusão social, pois diminui diferenças, por meio de pesquisa e desenvolvimento de produtos universais. A deficiência, vale lembrar, é marcada pela perda de uma das funções do ser humano, seja ela física, psicológica ou sensorial. O indivíduo pode, assim, ter uma deficiência, mas isso não significa necessariamente que ele seja incapaz; a incapacidade poderá ser minimizada quando o meio lhe possibilitar acessos. (SCHIRMER; BROWNING; BERSCH e MACHADO, 2007, p. 21). O design ergonômico, um segmento do design, apresenta metodologias que aprimoram o desenvolvimento do projeto do produto, através da interface homem-tecnologia.

Em um projeto, a interface homem-máquina depende de sua configuração. Normalmente a interface é definida pela quantidade e variedade do sistema de entradas e saídas oferecidas, cuja arquitetura implica em diversas configurações e projetos em que as interfaces são baseadas. Dessa forma, podem-se classificar as interfaces de acordo com a modalidade: Sistemas Unimodais e Multimodais.

Sistemas Unimodais: possui apenas um canal de comunicação, a relação entrada e saída é única. Conforme a interação, esses sistemas podem ser baseados em categorias denominadas:

- Visão: uma categoria ampla devida à quantidade de aplicações. Os pesquisadores têm a disponibilidade de trabalhar com vários problemas e abordagens, resultando em diversos aspectos do feedback humano, como expressões faciais, movimentos, gestos, e outros;

- Áudio: essa interação entre homem e máquina são mais confiáveis e úteis. A interface ocorre com a locução, análise da emoção pelo áudio; definição de sons ou ruídos (suspiro, respiração, riso, etc.), música e outros;

- Sensores: uma categoria que envolve várias áreas e de aplicações, que contém um sensor físico comum para possibilitar a interação homem-máquina, como o uso de teclado, joysticks, sensores de movimento e digitalizadores, sensores de cheiro, de gosto, entre outros.

Sistemas multimodais: envolve diversos canais de comunicação, cuja relação de entrada e saída é de $\mathrm{n}$ para $\mathrm{m}$. Os tipos de canais referem-se comunicação humana, por meio dos sentidos: visão, audição, tato, olfato, e paladar.

Essa interface atua como uma mediadora de interação entre o homem e a máquina. Pode ter diversas combinações de modos de entrada como expressões faciais, fala, gestos e movimento dos olhos.

Os vários projetos de CRIs denotam uma característica comum: a preocupação com a adaptabilidade ao usuário e a segurança, conforme as necessidades individuais. Além disso, 0 sistema deve ser interativo, controlado pelo cadeirante e o software da CRI. Dessa forma, a interface homem-máquina é fundamental na pesquisa e desenvolvimento de uma CRI, que é uma Tecnologia Assistiva. 


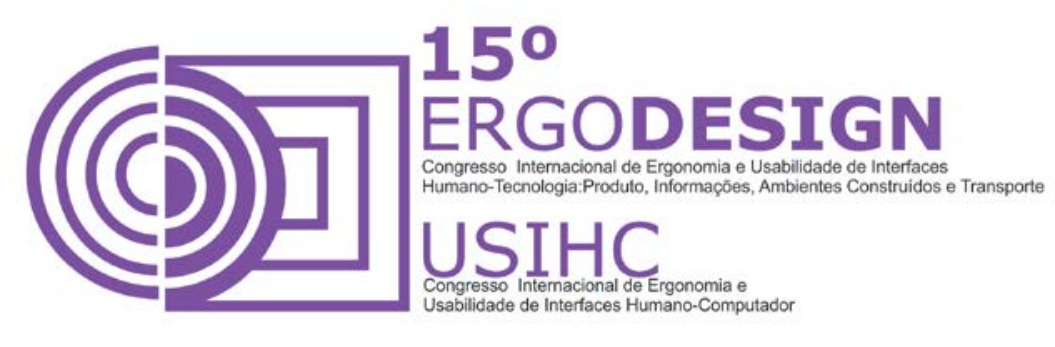

\subsection{O Sistema Arduino e suas contribuições para um design acessível}

O Arduino é um sistema advindo do conceito de hardware e Open Source que surgiu em 2005, na Itália, com o intuito de criar um dispositivo para o controle de projetos desenvolvidos com o custo menor. Uma plataforma de computação física (sistemas digitais que permitem criar sistemas que respondam com ações físicas), baseado em uma placa de entrada/saída e de programação. O Arduino é o que chamamos de plataforma de computação física ou embarcada, ou seja, um sistema que pode interagir com seu ambiente por meio de hardware e software. (MCROBERTS, 2011, p. 22).

Pode ser usado no desenvolvimento de artefatos interativos stand-alone ou conectados ao computador através de programas como o Pure Data, Adobe Flash, Processing, Max/MSP ou SuperCollider. O Arduino pode ser utilizado para desenvolver objetos interativos independentes, ou pode ser conectado a um computador, a uma rede, ou até mesmo à Internet para recuperar e enviar dados do Arduino e atuar sobre eles. Em outras palavras, ele pode enviar um conjunto de dados recebidos de alguns sensores para um site, dados estes que poderão, assim, ser exibidos na forma de um gráfico. (MCROBERTS, 2011, p. 23).

A placa do Arduino Uno, como mostra a Figura 2, não utiliza o chip FTDI, que conduz a USB para a serial. Utiliza um Atmega8U2, um conversor USB para serial, permitindo que o chip USB tenha seu firmware atualizado, assim o Arduino pode ser exibido em PC como outro dispositivo, como um mouse ou joystick. Uma plataforma de hardware livre, que utiliza micro controlador Atmel AVR de placa única, que possui linguagem de programação essencialmente em $\mathrm{C} / \mathrm{C}++$, para criar ferramentas acessíveis, com baixo custo, flexíveis e práticas.

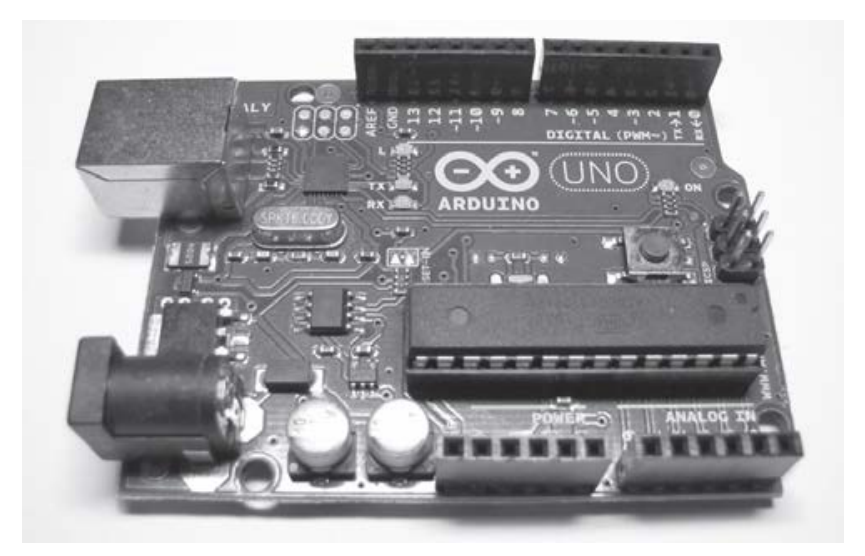

Figura 2. Arduino Uno. Fonte: McRobert (2011).

O hardware e o software do Arduino são de fonte aberta, dessa forma códigos, esquemas, projetos, entre outros podem ser utilizados livremente, por isso há muitas placas-clone disponíveis no mercado. O sistema também oferece o uso de shields (escudos), placas de 


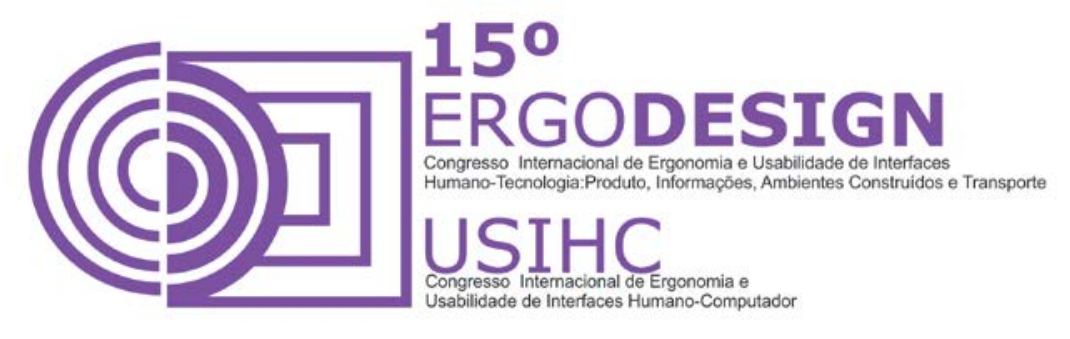

circuito que contém outros dispositivos como GPS, receptores, módulos de Ethernet, displays de LCD, e outros.

Para programar o Arduino deve ser utilizado o IDE do Arduino, um software para código na linguagem $C$. O IDE permite escrever um programa de computador, para upload do Arduino e a execução das instruções. Os programas escritos usando Arduino são chamados sketches, que permitem recortar/colar e procurar/substituir texto.

Pode-se ainda adicionar ao firmware, um recurso a CRI que permite o desvio de obstáculos. Firmware conhecido como "software embarcado", um conjunto de instruções operacionais com a possibilidade de programa-las ao hardware de equipamentos eletrônicos.

Nesse contexto, podemos afirmar a contribuição do Arduino no aprimoramento de tecnologias assistivas por possibilitar o desenvolvimento de objetos interativos independentes, ou conectados a um computador. Tais recursos propiciam ao portador de lesão medular maior qualidade de vida, com independência e segurança.

A acessibilidade é um direito regido por lei, porém ainda há necessidade de pesquisas e objetos para que deficientes físicos consigam realizar suas atividades sem dificuldade de execução, isso ocorre principalmente quando utilizam cadeiras tradicionais, que exigem grande esforço dos braços para a locomoção.

Esse estudo procurou enfatizar um dos recursos que permite melhorar a capacidade de locomoção, a cadeira de rodas inteligente, que contribui de forma significativa para aqueles que têm mobilidade reduzida.

A acessibilidade é uma condição que depende da ergonomia, do design e de outras áreas importantes. Dessa forma, torna-se um processo científico (exigindo diversas áreas do conhecimento) e social (dependência de políticas públicas e empresariais).

\section{REFERÊNCIAS BIBLIOGRÁFICAS}

ANTONELI, M. Prescrição de cadeira de rodas. In: TEIXEIRA, E. et al. (Ed.). Terapia ocupacional na reabilitação física. Rocca. São Paulo, 2003.

BITTENCOURT, G. Inteligência Artificial: ferramentas e teorias. UFSC. Santa Catarina, 2006.

BRAGA, R. A. M. Plataforma de Desenvolvimento de Cadeiras de Rodas Inteligentes. Faculdade de Engenharia da Universidade do Porto. Departamento de Engenharia Informática. Porto, 2010

IBGE. Instituto Brasileiro de Geografia e Estatística. Censo Demográfico 2010. Características gerais da população, região e pessoas com deficiência. Disponível em: ftp://ftp.ibge.gov.br/Censos/Censo Demografico 2010/Caracteristicas Gerais Religiao Deficiencia/carac teristicas religiao deficiencia.pdf Acesso em 11 set. 2013.

IIDA, I. Ergonomia: projeto e produção. Edgard Blücher. São Paulo, 1990. 


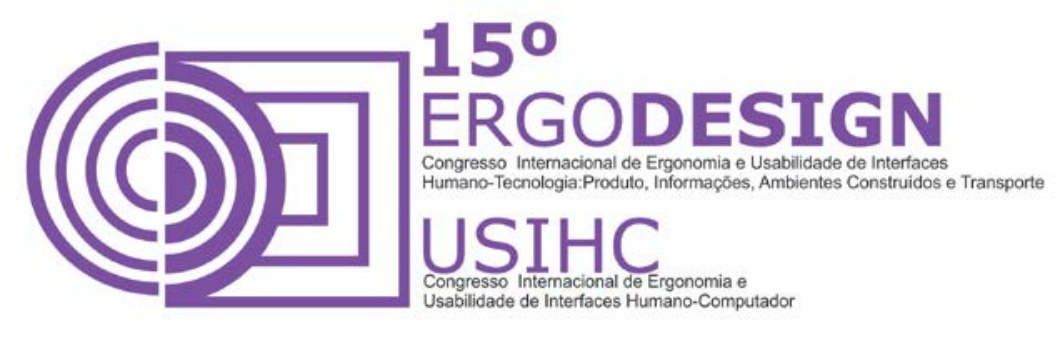

JORDAN, P. W. Human factors for pleasure in product use. Applied Ergonomics, vol. 29, n. 1, pp. 2533. 1998.

LOBACH, B. Design industrial: bases para a configuração dos produtos industriais. Blucher. São Paulo, 2001.

MELLO, M. Tecnologia assistiva. In: GREVE, J. M. D.; AMATUZZI, M. M. Medicina de reabilitação aplicada à ortopedia e traumatologia. São Paulo: Manole, 1997. RCHESAN, M. Sistema de monitoramento residencial utilizando a plataforma arduino. Santa Maria. 2012. ROCHA, E. F.; CASTIGLIONI, M. C. Reflexões sobre recursos. Rev. Ter. Ocup. Univ. v. 16, n. 3, p. 97-104, set./dez., São Paulo, 2005.

REZENDE, S.O. Sistemas inteligentes: fundamentos e aplicações. São Paulo: Manole. 2005.

RUSSEL, S.; NORVIG, P. Inteligência Artificial. Campus. Rio de Janeiro, 2004.

SANTOS, E. S. dos. Ergonomia e Acessibilidade: um estudo de caso sobre os problemas potenciais na concepção de sistema de locomoção para atividades diárias na cidade de Manaus. Projética. v.4, n.1, p. 121-136, Jan./Jun. Londrina, 2013.

SCHIRMER, C. R., BROWNING, N., BERSCH, R., MACHADO, R. Portal Mec. Atendimento Educacional Especializado: Deficiência Física. Disponível em: http://portal.mec.gov.br/seesp/arquivos/pdf/aee df.pdf Acessado em 28 set. 2013.

SILVEIRA E. Da. Revista Pesquisa FAPESP. Mobilidade ampliada: Sistema permitirá aos portadores de paralisias graves comandarem cadeira de rodas com músculos da face. Ed. 208. Jun/2013. Disponível em: http://revistapesquisa.fapesp.br/2013/06/05/mobilidade-ampliada/ Acesso em 01 jul. 2014. 